\title{
Dietas para emagrecimento publicadas em um magazine direcionado ao público feminino: análise do conteúdo nutricional
}

\author{
Nutritional analysis of weight loss diets published in a women's magazine \\ Rayanne Santos Floriano ${ }^{1} \bowtie$, Caryna Eurich Mazur², Kélin Schwarz³, Simone Carla Benincá2 \\ Tatiane Winkler Marques Machado ${ }^{3}$ \\ ${ }^{1}$ Faculdade Campo Real (FCR). Guarapuava, PR. \\ ${ }^{2}$ Colegiado de Nutrição. Faculdade Campo Real (FCR). Guarapuava, PR. \\ ${ }^{3}$ Departamento de Nutrição. Universidade Estadual do Centro-Oeste (UNICENTRO). Guarapuava, PR.
}

\section{RESUMO}

Objetivos: Avaliar nutricionalmente dietas veiculadas em uma revista não científica direcionada ao público feminino e comparar o seu conteúdo com as recomendações dietéticas atuais de macronutrientes e micronutrientes para mulheres adultas.

Métodos: Foram selecionados os 24 números publicados em 2013 e 2014 pela revista feminina de circulação nacional da maior circulação nesse período, e analisadas as dietas para emagrecimento veiculadas nesses números. A composição nutricional foi calculada pelo software Avanutri $^{\circledR}$ e comparada com as recomendações atuais do Institute of Medicine para macro e micronutrientes. As dietas foram divididas em dois grupos: de muita restrição calórica $(\leq 1100 \mathrm{kcal})$ e de pouca restrição calórica $(>1100 \mathrm{kcal})$. A análise compreendeu a comparação entre as dietas e as recomendações atuais e a comparação entre os dois grupos.

Resultados: Foram analisadas 20 dietas, as quais apresentavam um valor calórico médio de 1227,83 $\pm 261,54$ kcal. Todas as dietas estavam inadequadas em vitamina D (média: 2,09 $\pm 1,16$ ), ácido fólico (média: 109,26 $\pm 41,19$ ), ferro (média: 11,35 $\pm 3,06$ ), cálcio (média: 532,23 $\pm 133,56$ ) e zinco (média: 4,23 2,11). Quando comparados os dois grupos de dietas, as dietas de muita restrição calórica indicavam menores teores de vitamina $C(p=0,011)$, ferro $(p=0,051)$, cálcio $(p=0,022)$ e zinco $(p=0,033)$. Em relação aos macronutrientes, a maioria das dietas estava de acordo com os valores recomendados. Em relação às fibras, todas as dietas ficavam abaixo dos valores recomendados. A maioria das dietas com inadequação de macro e micronutrientes pertencia ao grupo de muita restrição calórica.

Conclusões: A maioria das dietas recomendadas pela revista analisada apresentava um reduzido aporte de energia e nutrientes, incluindo vitaminas e minerais importantes para mulheres em idade fértil. As dietas de muita restrição calórica apresentavam mais frequentemente valores nutricionais inadequados. Este resultado reforça a importância de que dietas sejam individualizadas e acompanhadas por profissional habilitado.

DESCRITORES: dieta; regime alimentar; perda de peso; recomendações nutricionais.

\section{ABSTRACT}

Aims: To nutritionally analyze diets published in a women's magazine and compare them with current dietary recommendations for macronutrient and micronutrient intake by adult women.

Methods: Twenty-four issues published in 2013 and 2014 by women's magazine with the largest circulation in that period were selected, and the weight-loss diets therein were assessed. The nutritional composition was calculated by the Avanutri ${ }^{\circledR}$ software and compared with the current recommendations from the Institute of Medicine for macronutrients and micronutrients. The diets were divided into two groups: large caloric restriction $(\leq 1,100 \mathrm{kcal})$ and slight caloric restriction $(>1,100 \mathrm{kcal})$.

Results: Twenty diets were analyzed, with an average caloric value of 1,227.83 $\pm 261.54 \mathrm{kcal}$. All diets were deficient in vitamin D (mean of $2.09 \pm 1.16$, folic acid (mean of 109.26 \pm 41.19 ), iron (mean of 11.35 \pm 3.06 ), calcium (mean of 532.23 \pm 133.56 ), and zinc (mean of 4.23 \pm 2.11 ). When both groups were compared, diets with large caloric restriction had lower concentrations of vitamin $C(p=0.011)$, iron $(p=0.051)$, calcium $(\mathrm{p}=0.022)$, and zinc $(\mathrm{p}=0.033)$. Most diets contained the recommended amount of macronutrients, but all of them were lacking in fibers. Most diets with inappropriate amounts of macronutrients and micronutrients were in the large caloric restriction group.

Conclusions: Most diets had a deficient supply of energy and nutrients, especially vitamins and minerals that are important for women of childbearing age. Diets with large caloric restriction often contained inappropriate nutritional values. This result reinforces the importance that diets be individualized and monitored by a qualified professional.

KEY WORDS: diet; weight loss; recommended dietary allowances. 
Abreviaturas: AMDR, acceptable macronutrients distribution range; DRI, dietary reference intakes; VCT, valor calórico total; RDA, recommended dietary allowance; IOM, Institute of Medicine.

\section{INTRODUÇÃO}

A mídia exerce papel organizador na construção e desconstrução de processos alimentares, fazendose presente entre o arsenal de produtos da indústria de emagrecimento. Inúmeras revistas sobre saúde e boa forma vendem em suas capas emagrecimento de forma rápida e o tão sonhado corpo das estrelas por meio de dietas. Aproveitando as preocupações com os padrões de beleza, a mídia veicula com frequência conceitos incorretos, seguindo modismos e impondo uma alimentação considerada "leve e inteligente", direcionada às pessoas bem-sucedidas, fazendo com que as leitoras de revistas se prendam a padrões inatingíveis e impulsionando distúrbios alimentares e de imagem corporal. Personalidades cada vez mais magras transformam-se em padrões estéticos a serem seguidos. Esse apelo pela busca do corpo ideal tem aumentado a propagação de dietas impróprias no âmbito nutricional, estimulando práticas alimentares e de estilo de vida não saudáveis. As mulheres são o principal alvo dessas imposições, que associam a aparência física ao sucesso profissional, independência e autonomia financeira [1-8].

Por outro lado, a pressão exercida pela publicidade do corpo perfeito pode gerar ansiedade e culpa em relação ao ato de comer, podendo levar a transtornos alimentares graves, como anorexia, bulimia, compulsão alimentar periódica e, até mesmo, a sobrepeso e obesidade. Comportamentos de supressão de refeições contribuem para a reserva de gordura e, consequentemente, para o aumento de peso. Nesse caso, os riscos de doenças associadas aumentam, como os de doenças crônicas não transmissíveis, responsáveis por $72 \%$ das causas de óbito no Brasil. Sobrepeso/obesidade já constituem um problema de saúde pública mundial [9-13].

Sabe-se que uma dieta inadequada pode resultar em deficiência tanto de macronutrientes quanto de micronutrientes, podendo prejudicar o funcionamento de órgãos, sistemas ou processos metabólicos do corpo humano [14-19]. Nas mulheres em idade fértil essas deficiências podem causar danos ainda maiores, considerando-se a função reprodutiva dessa faixa etária e as graves repercussões que podem advir da carência de determinados nutrientes [20-26].
Diante disso, o objetivo do presente estudo foi avaliar quantitativamente, sob o aspecto nutricional, as dietas de emagrecimento publicadas em uma revista não científica de circulação nacional direcionada ao público feminino, comparando-as com as recomendações dietéticas de macro e micronutrientes atualmente preconizadas para mulheres adultas.

\section{MÉTODOS}

Foram selecionadas as 24 edições publicadas entre janeiro de 2013 e dezembro de 2014 pela revista feminina de circulação nacional mais vendida nesse período, de acordo com o Instituto Verificador de Comunicação [27], com conteúdo e escopos principais de emagrecimento e boa forma física. Inicialmente, foram identificadas todas as dietas e/ou cardápios de emagrecimento ou manutenção de peso veiculadas nos exemplares. Não foram incluídas as edições que não continham indicação de dietas. Foram excluídos cardápios com alimentos específicos não encontrados na base de dados do programa utilizado para avaliação, assim como cardápios com menos de três dias de sugestão. Por cuidado ético, o nome da revista, bem como da editora, foram omitidos do estudo.

Para análise quantitativa das dietas foi utilizado o software AVANUTRI ${ }^{\circledR}$ versão 4.0 (Rio de Janeiro, Brasil) [28], considerando-se os valores descritivos para cada cardápio proposto pela revista. Foram analisados os seguintes dados: valor calórico total (kcal), carboidratos (\%), lipídeos (\%), proteínas (\%) e fibras dietéticas de acordo com Acceptable Macronutrients Distribution Range (AMDR) [29]; cálcio (mg), ferro (mg), ácido fólico (mg), zinco (mcg), vitamina D (mg), vitamina C (mg) e vitamina A (mcg), de acordo com Dietary Reference Intakes (DRI) [30-33] e colesterol segundo a Sociedade Brasileira de Cardiologia [34]. Sal, açúcar e edulcorantes foram considerados somente quando indicados na dieta. Uma vez citadas medidas caseiras (como colher de sopa, copo americano, prato de sobremesa) utilizou-se o equivalente em gramas, presente no próprio software de cálculo de dietas. Quando não apontados os tamanhos das porções, considerou-se uma unidade média ou padrão. Como critério para a escolha desses nutrientes avaliou-se a importância que os mesmos têm na saúde da mulher adulta, e o efeito do seu déficit ou excesso na saúde.

O padrão estabelecido foi para uma mulher de 19 a 50 anos com necessidade energética de $1800 \mathrm{kcal} /$ dia. Para determinação do valor energético diário total da 
dieta foi considerada a quantidade de energia fornecida por grama de carboidrato $(4 \mathrm{kcal} / \mathrm{g})$, proteína $(4 \mathrm{kcal} / \mathrm{g})$ e lipídeo $(9 \mathrm{kcal} / \mathrm{g})$. Para avaliação dos macronutrientes de acordo com AMDR foram considerados valores adequados de carboidrato entre $45 \%$ a $65 \%$ do valor calórico total (VCT) da dieta; proteínas entre 10\% a $35 \%$ e lipídeos de $20 \%$ a $35 \%$ do VCT; valores abaixo dos recomendados foram considerados insuficientes e, acima desses, considerados valores excessivos. Já para a determinação dos micronutrientes de acordo com DRI, foram adotados como ponto de corte os valores de Recommended Dietary Allowances (RDA), que considera a quantidade de nutriente adequada para 97-98\% da população. Quando não se obteve o valor de RDA (como para o cálcio e a vitamina D) consideraram-se os valores de Adequate Intake, que estimam os valores dietéticos recomendados quando não há estudos para determinar o valor de RDA [29-33].

Fibras foram consideradas adequadas quando ingeridas entre 25 a $30 \mathrm{~g} / \mathrm{dia}$ [16]. Colesterol abaixo de $300 \mathrm{mg} /$ dia é o ideal de acordo com a Sociedade Brasileira de Cardiologia [34]. As dietas foram divididas em dois grupos: dieta de muita restrição calórica ( $\leq 1100 \mathrm{kcal}$ por dia) e dieta de pouca restrição calórica (>1101 kcal por dia). Os dados foram analisados por meio de teste de análise de variância (ANOVA), teste de exato de Fischer e teste T de Student, utilizando o software IBM SPSS versão 22.0 [35].

\section{RESULTADOS}

Em 22 das 24 edições da revista publicadas nos dois anos em estudo havia indicação de dietas que atenderam aos critérios de inclusão, uma por cada edição. Dessas 22 dietas, duas foram excluídas, uma por conter alimentos específicos não encontrados na base de dados do programa e uma por incluir menos de três dias de sugestão. Assim, a amostra final foi constituída por 20 cardápios.

O valor calórico total médio das dietas foi de $1227,83 \pm 261,54 \mathrm{kcal}$ (bem abaixo do valor considerado como preconizado, descrito na metodologia). A maioria dos cardápios apresentou uma dieta de pouca restrição calórica: $60 \%$, versus $40 \%$ de muita restrição.

Foi possível observar que todas as dietas estavam inadequadas em vitamina D (média: 2,09 $\pm 1,16$ ), ácido fólico (média: 109,26 $\pm 41,19$ ), ferro (média: 11,35 $\pm 3,06$ ), cálcio (média: 532,23 $\pm 133,56$ ) e zinco (média: 4,23 $\pm 2,11$ ). A oferta média de colesterol nas dietas analisadas foi de $232,94 \mathrm{mg}$, sendo que em $12 \%$ das dietas o valor diário estava acima do considerado adequado $(<300 \mathrm{mg})$.

Menores teores de vitamina $\mathrm{C}$, ferro, cálcio e zinco foram apresentados nas dietas de muita restrição calórica em relação às dietas de pouca restrição calórica. Não foram observadas diferenças significativas no conteúdo dos demais micronutrientes e macronutrientes entre os dois grupos de dietas (Tabela 1).

Tabela 1. Comparação entre valores diários médios dos nutrientes das 20 dietas analisadas, publicadas por uma revista não científica direcionada ao público feminino, conforme a restrição calórica e o valor preconizado na literatura.

\begin{tabular}{|c|c|c|c|c|c|}
\hline Nutrientes & $\begin{array}{c}\text { Valor } \\
\text { preconizado* }\end{array}$ & $\begin{array}{l}\text { Todas as dietas } \\
n=20 \\
\text { (Média } \pm \text { DP) }\end{array}$ & $\begin{array}{c}\text { Dieta de muita restrição } \\
\text { calórica: } \leq 1100 \text { kcal } \\
n=8 \\
\text { (Média } \pm \text { DP) }\end{array}$ & $\begin{array}{c}\text { Dieta de pouca restrição } \\
\text { calórica: }>1100 \text { kcal } \\
n=12 \\
\text { (Média } \pm \text { DP) }\end{array}$ & $\mathbf{p}$ \\
\hline Calorias & 1800 & $1227,83 \pm 261,54$ & $1055,25 \pm 65,01$ & $1438,76 \pm 257,23$ & $0,002^{\dagger}$ \\
\hline Carboidrato (\%) & $45-65$ & $49,84 \pm 6,94$ & $48,74 \pm 8,75$ & $51,21 \pm 3,83$ & $0,196 * *$ \\
\hline Proteína (\%) & $10-35$ & $24,62 \pm 4,12$ & $24,21 \pm 4,76$ & $25,13 \pm 3,38$ & $0,970 * *$ \\
\hline Lípídeo (\%) & $20-35$ & $25,54 \pm 4,82$ & $27,07 \pm 5,83$ & $23,67 \pm 2,36$ & $0,087 * *$ \\
\hline Vitamina A (mcg) & 700 & $1298,67 \pm 714,31$ & $1156,17 \pm 753,72$ & $1472,83 \pm 663,08$ & $0,239^{\dagger}$ \\
\hline Vitamina D (mg) & 5 & $2,09 \pm 1,16$ & $1,85 \pm 1,01$ & $2,37 \pm 1,33$ & $0,327^{* *}$ \\
\hline Vitamina C (mg) & 75 & $172,21 \pm 140,24$ & $104,19 \pm 38,79$ & $255,35 \pm 175,18$ & $0,011 * *$ \\
\hline Ácido fólico (mcg) & 400 & $109,26 \pm 41,19$ & $114,39 \pm 46,42$ & $102,99 \pm 35,42$ & $0,553^{+}$ \\
\hline Ferro (mg) & 18 & $11,35 \pm 3,06$ & $10,16 \pm 2,31$ & $12,79 \pm 3,36$ & $0,051^{\dagger}$ \\
\hline Cálcio (mg) & 1000 & $532,23 \pm 133,56$ & $473,01 \pm 126,81$ & $604,62 \pm 107,44$ & $0,022^{\dagger}$ \\
\hline Zinco (mg) & 8 & $4,23 \pm 2,11$ & $3,34 \pm 1,45$ & $5,31 \pm 2,35$ & $0,033^{+}$ \\
\hline Colesterol (mg) & $\downarrow 300$ & $232,94 \pm 65,82$ & $223,87 \pm 54,97$ & $244,01 \pm 79,11$ & $0,511^{\dagger}$ \\
\hline
\end{tabular}

* Conforme descrito na metodologia.

** Referente ao teste de Wilcoxon-Mann-Whitney.

+ Teste T de Student.

DP desvio padrão. 
$\mathrm{Na}$ análise do percentual de adequação das dietas avaliadas conforme recomendações para fibras e macronutrientes, a insuficiência na quantidade de fibras foi observada nas 20 dietas (100\%). Em relação aos carboidratos, $16(80 \%)$ das dietas apresentaram os valores adequados, entre $45 \%$ a $65 \%$ do VCT da dieta, enquanto quatro $(20 \%)$ encontravam-se abaixo do recomendado. O teor de lipídeos foi adequado em $17(85 \%)$ das dietas de acordo com os valores preconizados, em duas (10\%) abaixo do recomendado e em uma (5\%) acima do indicado. Quanto às proteínas, as 20 dietas $(100 \%)$ atendiam ao valor preconizado (Figura 1).

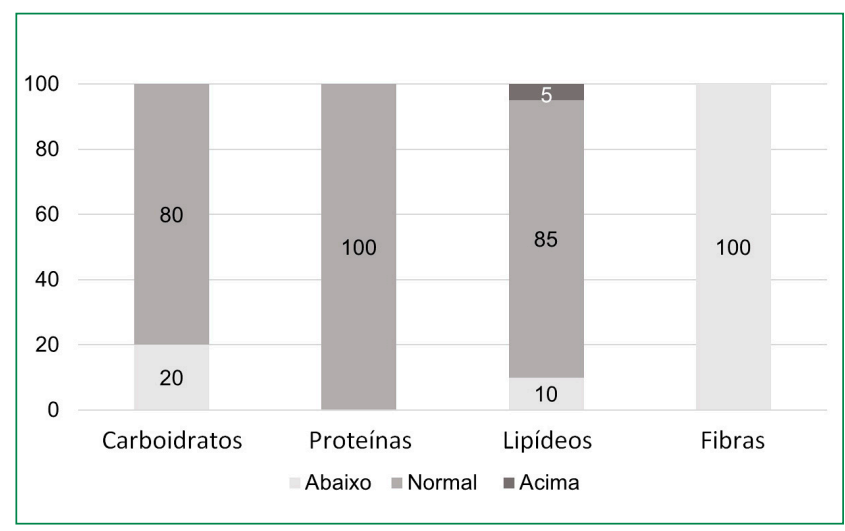

Figura 1. Percentuais de adequação de macronutrientes e fibras nas 20 dietas analisadas, publicadas por uma revista não científica direcionada ao público feminino.
Quanto à comparação entre restrição calórica e adequação de macronutrientes de acordo com os grupos de dietas, observou-se que mais dietas com inadequação de macronutrientes encontrava-se no grupo de muita restrição calórica. Em relação ao teor de fibras, ambos os grupos possuíam 100\% das dietas com valores abaixo do adequado. Nestes quesitos as diferenças entre os grupos não foram estatisticamente significativas (Tabela 2).

\section{DISCUSSÃO}

Este estudo identificou que todas as dietas analisadas estavam abaixo dos valores calóricos recomendados para mulheres na faixa etária alvo (1800 kcal/dia). Os resultados são semelhantes aos encontrados por Farias et al. [14] que, ao analisarem a composição nutricional de dietas da moda publicadas em revistas, observaram uma média de valor energético de $1182 \mathrm{kcal} / \mathrm{dia}$. Outros estudos semelhantes também encontraram valores calóricos abaixo dos recomendados [15-17].

Di Vetta et al. [36] demonstraram que dietas hipocalóricas apresentam-se ineficientes a longo prazo para a perda de peso. Em seu estudo, cerca de $80 \%$ das participantes recuperaram o peso após um mês de dieta, e apenas 1\% manteve o peso alcançado após um ano [36]. Além disso, podem surgir sintomas causados por dietas muito restritivas, como diminuição do débito cardíaco e da pressão arterial, queda de cabelo, fadiga, dores de cabeça, anemia e irregula-

Tabela 2. Comparação entre restrição calórica dicotomizada entre muita e pouca restrição e adequação de macronutrientes e fibras nas 20 dietas analisadas, publicadas por uma revista não científica direcionada ao público feminino.

\begin{tabular}{|c|c|c|c|c|}
\hline & $\begin{array}{c}\text { Todas as dietas } \\
n=20 \\
n(\%)\end{array}$ & $\begin{array}{l}\text { Dieta de muita restrição } \\
\text { calórica: } \leq 1100 \text { kcal } \\
n=8 \\
n(\%)\end{array}$ & $\begin{array}{l}\text { Dieta de pouca restrição } \\
\text { calórica: }>1100 \text { kcal } \\
n=12 \\
n(\%)\end{array}$ & $\mathbf{p}^{*}$ \\
\hline \multicolumn{5}{|l|}{ Carboidrato } \\
\hline Abaixo & $4(20)$ & $2(25)$ & $2(16,7)$ & 0,375 \\
\hline Adequado & $16(80)$ & $6(75)$ & $10(83,3)$ & \\
\hline \multicolumn{5}{|l|}{ Proteína } \\
\hline Adequado & 20 (100) & $8(100)$ & $12(100)$ & - \\
\hline \multicolumn{5}{|l|}{ Lipídeo } \\
\hline Abaixo & $2(10)$ & $2(25)$ & - & 0,09 \\
\hline Adequado & $17(85)$ & $5(62,5)$ & $12(100)$ & \\
\hline Acima & $1(5)$ & $1(12,5)$ & - & \\
\hline \multicolumn{5}{|l|}{ Fibras } \\
\hline Abaixo & $20(100)$ & $8(100)$ & $12(100)$ & - \\
\hline
\end{tabular}

* Teste exato de Fisher. 
ridades menstruais $[15,18]$. Deve-se salientar que dietas com oferta menor de $1200 \mathrm{kcal} /$ dia não suprem a necessidade mínima de micronutrientes para mulheres adultas, bem como a taxa de metabolismo basal [4].

Quanto à distribuição de macronutrientes, constatou-se que todas as dietas eram normoproteicas, corroborando com os resultados encontrados por Farias et al. [14] e Contão et al. [37], em que a maioria dos cardápios apresentou adequação de proteínas. Já outros estudos encontraram que a maioria das dietas eram hiperproteicas $[38,39]$. Essa diferença pode ser explicada pelo desequilíbrio entre os padrões utilizados para verificar a normalidade dos teores de proteínas. Enquanto alguns estudos consideram os valores preconizados pelo Institute of Medicine (IOM) (10\% a $35 \%$ ) [29], outros utilizam valores que variam de $10 \%$ a $15 \%$, abrangendo uma menor faixa para o aceitável de proteínas na dieta.

Uma dieta com maior teor de proteínas tende a promover o emagrecimento, em razão da saciedade provocada pelo efeito termogênico maior e mais demorado das proteínas em relação aos lipídeos e carboidratos. Entretanto, apesar de alguns estudos evidenciarem que uma maior proporção de proteínas na dieta favorece a perda de peso, esse efeito não é totalmente elucidado. Além disso, uma vez que a maior parte da proteína recomendada é geralmente de origem animal, pode haver aumento no consumo de gorduras saturadas e de colesterol e menor consumo de fibras, podendo resultar em doenças correlatas, como as cardiovasculares. Ingestão excessiva de proteína animal pode também levar à hipercalciúria, ocasionada por uma maior reabsorção óssea e diminuição da reabsorção tubular renal do cálcio [19,40-42].

Mesmo com a maioria das dietas apresentando valores adequados de carboidratos, $20 \%$ das dietas analisadas no presente estudo continham valores inferiores ao recomendado, corroborando com os resultados encontrados por Pacheco et al. [16], ao analisarem 30 dietas em publicações de revistas não científicas. Em dietas restritas em carboidratos, as reservas de glicogênio muscular e hepático são mobilizadas, processo metabólico que acarreta utilização de água. Logo, devido à diminuição das reservas de glicogênio, a perda de peso corporal devese à perda de água, e o peso é recuperado tão logo a ingestão de carboidratos seja retomada [18].

Nas dietas analisadas houve variação no teor de lipídeos, sendo que a maioria estava dentro dos valores preconizados pela DRI [29]. Lima et al. [4] também verificaram uma maioria de dietas normolipídicas. Apesar de os lipídeos serem nutrientes que de- sempenham papel energético, hormonal e estrutural no organismo, uma dieta rica em lipídeos favorece o desenvolvimento de obesidade e outras doenças [14]. Stringhini et al. [43] demonstraram que uma dieta hiperlipídica promove inicialmente a redução de peso devido ao seu maior poder de saciedade, porém a adesão tende a ser curta, e o peso é recuperado após o retorno a uma dieta equilibrada.

Dietas hipolipídicas também merecem atenção por poderem interferir na absorção de vitaminas lipossolúveis, ocasionar inadequação na ingestão de ácidos graxos essenciais e levar a baixos níveis de colesterol total e de colesterol ligado a lipoproteínas de alta densidade, entre outros prejuízos $[44,45]$. O colesterol é importante para a síntese de vitamina D e de hormônios como cortisol, aldosterona e estradiol. Entretanto, colesterol em excesso pode favorecer o desenvolvimento de doenças cardiovasculares [34]. Assim, enfatiza-se a importância do equilíbrio na composição lipídica da dieta.

Em relação às fibras, todas as dietas analisadas apresentaram valores inferiores a $25 \mathrm{~g} / \mathrm{dia}$, valor recomendado pelo IOM [29], concordando com as pesquisas de Lima et al. [4] e Matias [17], em que 100\% das dietas estavam inadequadas ao valor recomendo de fibras, e ainda ao de Pacheco et al. [16], em que somente uma dieta estava adequada à quantidade diária de fibra. De acordo com o Guia Alimentar para a População Brasileira [39], a ausência de fibras na dieta deve-se à falta ou presença limitada de alimentos in natura, devendo-se estimular a diminuição dos alimentos ultraprocessados, pela pequena quantidade de fibras, e o consumo adequado e variado de alimentos de origem vegetal, que tendem a ser ricos nesse nutriente $[47,48]$.

Devido às restrições calóricas, que trazem como consequência a menor oferta de micronutrientes, os teores de vitamina $\mathrm{D}$, ácido fólico, cálcio, ferro e zinco apresentaram-se abaixo das necessidades diárias recomendadas, em maior proporção no grupo de muita restrição calórica, resultado semelhante ao encontrado por Perinazzo et al. [7].

Das 20 dietas analisadas, cinco possuíam valores abaixo do recomendado para vitamina $\mathrm{A}$, resultado que se assemelha ao encontrado pelo estudo de Matias [17], com a mesma quantidade de dietas verificadas, no qual sete dietas apresentaram inadequação em relação à vitamina $\mathrm{A}$. A deficiência prolongada dessa vitamina pode acarretar alterações oculares, entre outras disfunções [37]. Em mulheres em idade fértil a deficiência de vitamina $\mathrm{A}$, mesmo sem resultar em sintomas, pode levar a riscos obstétricos, já que essa vitamina tem papel importante na reprodução, no 
crescimento e desenvolvimento do feto, crescimento tecidual materno e reserva hepática fetal [20-22].

Observou-se que $80 \%$ das dietas compreendiam níveis adequados de vitamina $\mathrm{C}$, resultado semelhante ao observado por Abreu et al. [49] em estudo que avaliou a adequação de micronutrientes em dietas voltadas para o público feminino, resultando em $82 \%$ das dietas adequadas em relação a oferta de vitamina $C$.

Considerando à vitamina $\mathrm{D}$, quase todas as dietas (95\%) estavam inadequadas, não contendo uma oferta suficiente da mesma, resultado próximo ao de Matias et al. [50], que observaram um total de $92,8 \%$ de dietas inadequadas para vitamina $\mathrm{D}$. A carência de vitamina D está relacionada com doenças autoimunes, como diabetes insulinodependente, artrite reumatoide, esclerose múltipla e doença inflamatória intestinal [23].

A oferta de folato era insuficiente em $100 \%$ das dietas analisadas, corroborando com os resultados obtidos por Carvalho et al. [31] em que nenhuma das dietas alcançou os valores preconizados. $\mathrm{O}$ ácido fólico é de extrema importância em mulheres em idade fértil, por ser visto não somente como um nutriente necessário na prevenção da anemia megaloblástica na gravidez, como também uma vitamina essencial na saúde reprodutiva [24]. Seu aporte adequado no período pré-natal não só reduz os riscos de defeitos no tubo neural na criança, como pode estar associado a um menor risco de distúrbios do espectro autista [25].

A deficiência de ferro é a causa mais comum de anemia nas mulheres em idade fértil, sendo associada às perdas menstruais e à gestação, e potencializada se o aporte não for suficiente para sua reposição [51]. A quantidade média insuficiente de ferro estimada nas dietas analisadas neste estudo também foi encontrada na pesquisa de Abreu et al. [49], em que as dietas analisadas proporcionavam uma oferta ainda menor de ferro.

Avaliando o cálcio, nenhuma dieta atingiu a adequação, assim como encontrado por Abreu et al. [49] ao analisarem dietas genéricas publicadas pela imprensa leiga. $O$ cálcio tem papel significativo na formação dos ossos e dentes [37,39].

Somente uma dieta atingiu o valor preconizado para zinco. Matias et al. [50] em sua análise de dietas para perda de peso, encontraram apenas três entre quatorze dietas dentro do valor recomendado. Leão et al. [52] demostraram a existência de relação entre o zinco e a leptina, hormônio responsável pela saciedade. A deficiência prolongada de zinco pode levar a transtornos como anorexia e intolerância à glicose e, na criança, pode provocar déficit de crescimento e atraso no desenvolvimento [26].

As limitações encontradas no presente estudo incluem a falta de porcionamento exato em muitos cardápios, tais como "salada a vontade", "uma taça", "um prato", os quais podem ter subestimado ou superestimado os resultados obtidos. Esse dado é mais um fator que demonstra que a adoção desse tipo de dieta resulta em diferentes interpretações, por não haver uma orientação individual e o esclarecimento de dúvidas. No entanto, os cálculos foram realizados de modo uniforme e padronizado em relação ao alimento e quantidade, utilizando porções de tamanho médio quando não especificadas nas dietas, a fim de minimizar erros.

$\mathrm{O}$ reduzido aporte calórico e de nutrientes das dietas analisadas, principalmente de vitaminas e minerais, mostra-se como um possível risco à saúde de mulheres em idade fértil, em que certos nutrientes são essenciais. É preocupante o fato de tais dietas não seguirem preceitos básicos da nutrição, incluindo avaliação nutricional, recomendações individualizadas e adequada técnica dietética, fundamentais para o sucesso do tratamento nutricional. Os resultados deste estudo alertam para o perigo de seguir dietas preconizadas em revistas leigas ou, mesmo, em qualquer veículo de divulgação, sem uma orientação profissional.

\section{NOTA}

Declaração de conflitos de interesse

Os autores declaram não haver conflitos de interesse relevantes ao conteúdo deste estudo.

\section{REFERÊNCIAS}

1. Serra GMA, Santos EM. Saúde e mídia na construção da obesidade e do corpo perfeito. Ciênc Saúde Colet. 2003;8(3):691701. http://dx.doi.org/10.1590/S1413-81232003000300004

2. Chaud DMA, Marchioni DML. Nutrição e mídia: uma combinação às vezes indigesta. Hig Alimentar. 2004;18(116-117):18-22.

3. Santana HMM, Mayer MDB, Camargo KG. Avaliação da adequação nutricional das dietas para emagrecimento veiculadas pela internet. ConScientia e Saúde. 2003;(2):99-104. 
4. Lima KVG, Bion FM, Lima CR, Nascimento E, Albuquerque CG, Chagas MHC. Valor nutricional de dietas veiculadas em revistas não científicas. Rev Bras Promoç Saúde. 2010;23(4):349-57.

5. Saikali CJ, Soubhia CS, Scalfaro BM, Cordás TA. Imagem corporal nos transtornos alimentares. Rev Psiq Clin. 2004;31(4): 164-6. http://dx.doi.org/10.1590/S0101-60832004000400006

6. Bosi MLM, Raggio LR, Morgado CMC, Costa MLSS, Carvalho RJ. Autopercepção da imagem corporal entre estudantes de nutrição: um estudo no município do Rio de Janeiro. J Bras Psiquiatr. 2006;55(2):108-13. http://dx.doi.org/10.1590/S004720852006000200003

7. Perinazzo C, Almeida JC. Composição nutricional de dietas para emagrecimento divulgadas em revistas não científicas. Rev HCPA. 2010;30(3):233-40.

8. Serra GMA. Saúde nutrição na adolescência: o discurso sobre dietas na revista Capricho [dissertação]. [Rio de Janeiro]: Escola Nacional de Saúde Pública da Fundação Oswaldo Cruz; 2001. 137 p.

9. Souto S, Ferro-Bucher, JSN. Práticas indiscriminadas de dietas de emagrecimento e o desenvolvimento de transtornos alimentares. Rev Nutr. 2006;19(6):693-704. http://dx.doi.org/10.1590/S1415-52732006000600006

10. Proença RPC. Alimentação e globalização: algumas reflexões. Ciênc Cult. 2010;62(4):43-7.

11. Malta DC, Morais Neto OL, Silva Junior JB. Apresentação do plano de ações estratégicas para o enfrentamento das doenças crônicas não transmissíveis no Brasil, 2011 a 2022. Epidemiol Serv Saúde. 2011;20(4):425-38. http://dx.doi.org/10.5123/ S1679-49742011000400002

12. World Health Organization. Global status report on noncommunicable diseases 2014. Geneva: WHO; 2014.

13. Secretaria de Vigilância em Saúde, Ministério da Saúde. Vigitel Brasil 2014. Vigilância de fatores de risco e proteção para doenças crônicas por inquérito telefônico. Brasília: Ministério da Saúde; 2015.

14. Farias SJSS, Fortes RC, Fazzio DMG. Análise da composição nutricional de dietas da moda divulgadas por revistas não científicas. Nutrire Rev Soc Bras Aliment. 2014;39(2):196-202. http://dx.doi.org/10.4322/nutrire.2014.018

15. Willhelm FF, Oliveira RB, Coutinho VF. Composição nutricional de dietas para emagrecimento publicadas em revistas não científicas: comparação com as recomendações dietéticas atuais de macronutrientes. Nutrire Rev Soc Bras Aliment. 2014;39(2):179-86. http://dx.doi.org/10.4322/nutrire.2014.016

16. Pacheco CQ, Oliveira MAM, Stracieri APM. Análise nutricional de dietas publicadas em revistas não científicas destinadas ao público feminino. Rev Dig Nutr. 2009;3(4):e346-61.

17. Matias MO. Dietas da moda: os riscos nutricionais que podem comprometer a homeostase [Trabalho de conclusão de curso]. [Vitória]: Faculdade Católica Salesiana do Espírito Santo; 2014. 96 p.

18. Betoni F, Zanardo VPS, Ceni GC. Avaliação de utilização de dietas da moda por pacientes de um ambulatório de especialidades em nutrição e suas implicações no metabolismo. ConScientia e Saúde. 2010;9(3):430-40.

19. Layman DK, Evans E, Baum JI, Seyler J, Erickson DJ, Boileau RA. Dietary protein and exercise have additive effects on body composition during weight loss in adult women. J Nutr. 2005 Aug;135(8):1903-10.

20. Ramalho RA, Saunders C, Natalizi DA, Cardoso LO, Accioly E. Níveis séricos de retinol em escolares de 7 a 17 anos no município do Rio de Janeiro. Rev Nutr. 2004;17(4):461-8. http://dx.doi.org/10.1590/S1415-52732004000400006

21. Zimmermann MB. Interactions of vitamin A and iodine deficiencies: effects on the pituitary-thyroid axis. Int J Vitam Nutr Res. 2007 May;77(3):236-40. http://dx.doi.org/10.1024/0300-9831.77.3.236

22. Pereira ALF, Vidal TF, Constant PBL. Antioxidantes alimentares: importância química e biológica. Nutrire Rev Soc Bras Aliment Nutr. 2009;34(3):231-47.

23. Marques CDL, Dantas AT, Fragoso TS, Duarte ALBP. A importância dos níveis de vitamina D nas doenças autoimunes. Rev Bras Reumatol. 2010;50(1):67-80. http://dx.doi.org/10.1590/S0482-50042010000100007

24. Tamura T, Picciano MF. Folate and human reproduction. Am J Clin Nutr. 2006 May;83(5):993-1016.

25. Surén P, Roth C, Bresnahan M, Haugen M, Hornig M, Hirtz D, Lie KK, Lipkin WI, Magnus P, Reichborn-Kjennerud T, Schjølberg S, Davey Smith G, Øyen AS, Susser E, Stoltenberg C. Association between maternal use of folic acid supplements and risk of autism spectrum disorders in children. JAMA. 2013 Feb 13;309(6):570-7. http://dx.doi.org/10.1001/ jama.2012.155925

26. Mafra D, Cozzolino S. Importância do zinco na nutrição humana. Rev Nutr. 2004;17(1):79-87. http://dx.doi.org/10.1590/ S1415-52732004000100009

27. Instituto Verificador de Circulação - IVC. Relatórios de Auditoria (R. A.) [Internet]. [cited 2015 June 9]. Available from: http://ivcbrasil.org.br/online/SRelatoriosDeAuditoria.asp

28. Santana RI. Avanutri - Software de avaliação nutricional. Versão 4.0. Rio de Janeiro; 2009.

29. Institute of Medicine. Dietary reference intakes for energy, carbohydrate, fibre, fat, fatty acids, cholesterol, and protein and amino acids. Food and nutrition board. Washington DC: National Academy Press; 2005.

30. Institute of Medicine. Dietary reference intakes for calcium, phosphorus, magnesium, vitamin D and fluoride. Food and nutrition board. Washington DC: National Academy Press; 1997.

31. Institute of Medicine. Dietary reference intakes for thiamin, riboflavin, niacin, vitamin B6, folate, vitamin B12, pantothenic acid, biotin and choline. Food and nutrition board. Washington DC: National Academy Press; 1998. 
32. Institute of Medicine. Dietary reference intakes for vitamin A, vitamin K, arsenic, boron, chromium, copper, iodine, iron, manganese, molybdenum, nickel, silicon, vanadium and zinc. Food and nutrition board. Washington DC: National Academy Press; 2001.

33. Institute of Medicine. Dietary reference intakes for vitamin C, vitamin E, selenium and carotenoids. Food and nutrition board. Washington DC: National Academy Press; 2000.

34. Santos RD, Gagliardi ACM, Xavier HT, Magnoni CD, Cassani R, Lottenberg AMP, Casella Filho A, Araújo DB, Cesena FY, Alves RJ, Fenelon G, Nishioka SAD, Faludi AA, Geloneze B, Scherr C, Kovacs C, Tomazzela C, Carla C, Barrera-Arellano D, Cintra D, Quintão E, Nakandakare ER, Fonseca FAH, Pimentel I, Santos JE, Bertolami MC, Rogero M, Izar MCO, Nakasato M, Damasceno NRT, Maranhão R, Cassani RSL, Perim R, Ramos S. I Diretriz sobre o consumo de gorduras e saúde cardiovascular. Arq Bras Cardiol. 2013;100(1 Supl 3):1-40. http://dx.doi.org/10.5935/abc.2013S003

35. IBM Corp. Released 2013. IBM SPSS Statistics for Windows, Version 22.0. Armonk, NY: IBM Corp.; 2013.

36. Di Vetta V, Clarisse M, Giusti V. Hypocaloric diets: which ones to advise/avoid? Rev Med Suisse. 2005 Mar 23;1(12):818-22.

37. Contão BRC, Quintão LV, Silva SO. Avaliação das dietas para emagrecimento veiculadas por revistas femininas de baixo custo [Trabalho de conclusão de curso]. [Governador Valadares]: Faculdade de Ciências da Saúde da Universidade Vale do Rio Doce; 2009. 85 p.

38. Silva CSM, Kirsten VR. Avaliação da adequação de dietas publicadas por revistas não científicas. Discip Sci. 2014;15(1): 101-12.

39. Carvalho TBP, Faicari LM. Análise nutricional das dietas de emagrecimento veiculadas por revistas de circulação nacional. RBONE. 2014;8(43):4-15.

40. Paiva AC, Alfenas RCG, Bressan J. Efeitos da alta ingestão diária de proteínas no metabolismo. Rev Bras Nutr Clin. 2007;22(1):83-8

41. Pedrosa RG, Junior JD, Tirapegui J. Dieta rica em proteína na redução do peso corporal. Rev Nutr. 2009;22(1):105-11. http://dx.doi.org/10.1590/S1415-52732009000100010

42. Hession M, Rolland C, Kulkarni U, Wise A, Broom J. Systematic review of randomized controlled trials of low-carbohydrate vs. low-fat/low-calorie diets in the management of obesity and its comorbidities. Obes Rev. 2009 Jan;10(1):36-50. http://dx.doi.org/10.1111/j.1467-789X.2008.00518.x

43. Stringhini MLF, Silva JMC, Oliveira FG. Vantagens e desvantagens da dieta Atkins no tratamento da obesidade. Salusvita. 2007;26(2):257-68.

44. Polacow VO, Lancha Junior AH. Dietas hiperglicídicas: efeitos da substituição isoenergética de gordura por carboidratos sobre o metabolismo de lipídios, adiposidade corporal e sua associação com atividade física e com o risco de doença cardiovascular. Arq Bras Endocrinol Metab. 2007;51(3):389-400. http://dx.doi.org/10.1590/S0004-27302007000300006

45. Freedman MR, King J, Kennedy E. Popular diets: A scientific review. Obes Res. 2001 Mar;9 Suppl 1:1S-40S. http://dx.doi org/10.1038/oby.2001.113

46. Ministério da Saúde (BR). Guia alimentar para a população brasileira. 2a ed. Brasília (DF): Ministério da Saúde; 2014.

47. Steemburgo T, Dall'Alba V, Almeida JC, Zelmanovitz T, Gross JL, Azevedo MJ. Intake of soluble fibers has a protective role for the presence of metabolic syndrome in patients with type 2 diabetes. Eur J Clin Nutr. 2009 Jan;63(1):127-33. http://dx.doi.org/10.1038/sj.ejcn.1602902

48. Carvalho FS, Netto AP, Zach P, Sachs A, Zanella MT. Importância da orientação nutricional e do teor de fibras da dieta no controle glicêmico de pacientes diabéticos tipo 2 sob intervenção educacional intensiva. Arq Bras Endocrinol Metab. 2012;56(2):110-9.

49. Abreu ES, Paternez ACA, Chaud DMA, Valverde F, Gaze JS. Parâmetros nutricionais de dietas anunciadas na imprensa leiga destinada ao público masculino e feminino. Ciênc Saúde. 2013;6(3):206-13.

50. Matias D, Dias I. Popular diets: caracterização nutricional. Rev SPCNA. 2006;12(2):58-68.

51. Lopes SM, Freitas IR, Maciel MCC. Anemia ferropriva / ferropênica em gestantes: uma revisão integrativa de literatura. Rev Univ Vale Rio Verde. 2015;13(1):442-51. http://dx.doi.org/10.5892/ruvrd.v13i1.1989

52. Leão ALM, Santos LC. Consumo de micronutrientes e excesso de peso: existe relação? Rev Bras Epidemiol. 2012;15(1): 85-95. http://dx.doi.org/10.1590/S1415-790X2012000100008 